\title{
Supporting Information to "Advanced Deep Learning Spectroscopy of Scalogram Infused CNN Classifiers for Robust Identification of Post-Hypoxic Epileptiform EEG Spikes"
}

\author{
Hamid Abbasi ${ }^{1}$, Alistair J Gunn ${ }^{1}$, Charles P Unsworth ${ }^{1}$, and Laura Bennet ${ }^{1}$ \\ ${ }^{1}$ University of Auckland
}

October 26, 2020

\section{Appendix A}

Table S1. Results of the WS-CNN classifier for post-HI spike transient identification in experimental data (entire 6 hours - 13 layers)

\begin{tabular}{lll} 
Trained and validated on Sheep No. & No. of patterns in the Train and Validation Dataset & Tested on Sheep \\
$2,3,4,5,6,7$ & 4567 & 1 \\
$1,3,4,5,6,7$ & 4751 & 2 \\
$1,2,4,5,6,7$ & 4731 & 3 \\
$1,2,3,5,6,7$ & 3372 & 4 \\
$1,2,3,4,6,7$ & 4088 & 5 \\
$1,2,3,4,5,7$ & 4466 & 6 \\
$1,2,3,4,5,6$ & 4085 & 7 \\
\hline
\end{tabular}

Overall performance of the 13 layers WS-CNN in the entire 6 hours $\quad \mathbf{9 9 . 0 3} \pm \mathbf{1 . 6 6}$

Table S2. Results of the WS-CNN classifier for post-HI spike transient identification in experimental data (entire 6 hours -9 layers) 


\begin{tabular}{lll}
\hline Trained and validated on Sheep No. & No. of patterns in the Train and Validation Dataset & Tested on Sheep \\
$2,3,4,5,6,7$ & 4567 & 1 \\
$1,3,4,5,6,7$ & 4751 & 2 \\
$1,2,4,5,6,7$ & 4731 & 3 \\
$1,2,3,5,6,7$ & 3372 & 4 \\
$1,2,3,4,6,7$ & 4088 & 5 \\
$1,2,3,4,5,7$ & 4466 & 6 \\
$1,2,3,4,5,6$ & 4085 & 7 \\
\hline
\end{tabular}

Overall performance of the 9 layers WS-CNN in the entire 6 hours $\quad \mathbf{9 8 . 5 4} \pm \mathbf{1 . 4 3}$

Table S3. Results of the WS-CNN classifier for post-HI spike transient identification in experimental data (entire 6 hours -5 layers)

\begin{tabular}{lll} 
Trained and validated on Sheep No. & No. of patterns in the Train and Validation Dataset & Tested on Sheep \\
$2,3,4,5,6,7$ & 4567 & 1 \\
$1,3,4,5,6,7$ & 4751 & 2 \\
$1,2,4,5,6,7$ & 4731 & 3 \\
$1,2,3,5,6,7$ & 3372 & 4 \\
$1,2,3,4,6,7$ & 4088 & 5 \\
$1,2,3,4,5,7$ & 4466 & 6 \\
$1,2,3,4,5,6$ & 4085 & 7 \\
\hline
\end{tabular}

Overall performance of the 5 layers WS-CNN in the entire 6 hours $\quad \mathbf{9 7 . 7 0 \pm 1 . 9 9}$

\section{Appendix B}

Table S4. Results of the WF-CNN classifier for post-HI spike transient identification in experimental data (entire 6 hours - 11 layers) 


$\begin{array}{llll}\text { Trained and validated on Sheep No. } & \text { No. of patterns in the train dataset } & \text { Tested on Sheep No. } & \text { No. of pat } \\ 2,3,4,5,6,7 & 4567 & 1 & 443 \\ 1,3,4,5,6,7 & 4751 & 2 & 259 \\ 1,2,4,5,6,7 & 4731 & 3 & 279 \\ 1,2,3,5,6,7 & 3372 & 4 & 1638 \\ 1,2,3,4,6,7 & 4088 & 5 & 922 \\ 1,2,3,4,5,7 & 4466 & 6 & 544 \\ 1,2,3,4,5,6 & 4085 & 7 & 925\end{array}$

\section{Overall performance of the 11 layers WF-CNN in the entire 6 hours $\quad \mathbf{9 9 . 4 4 \pm 0 . 4 4}$}

Table S5. Results of the WF-CNN classifier for post-HI spike transient identification in experimental data (entire 6 hours -9 layers)

\begin{tabular}{llll} 
Trained and validated on Sheep No. & No. of patterns in the train dataset & Tested on Sheep No. & No. of pat \\
$2,3,4,5,6,7$ & 4567 & 1 & 443 \\
$1,3,4,5,6,7$ & 4751 & 2 & 259 \\
$1,2,4,5,6,7$ & 4731 & 3 & 279 \\
$1,2,3,5,6,7$ & 3372 & 4 & 1638 \\
$1,2,3,4,6,7$ & 4088 & 5 & 922 \\
$1,2,3,4,5,7$ & 4466 & 6 & 544 \\
$1,2,3,4,5,6$ & 4085 & 7 & 925 \\
\hline
\end{tabular}

\section{Overall performance of the 9 layers WF-CNN in the entire 6 hours $\quad \mathbf{9 9 . 3 3 \pm 0 . 3 6}$}

Table S6. Results of the WF-CNN classifier for post-HI spike transient identification in experimental data (entire 6 hours -7 layers)

\begin{tabular}{llll}
\hline Trained and validated on Sheep No. & No. of patterns in the train dataset & Tested on Sheep No. & No. of pat \\
$2,3,4,5,6,7$ & 4567 & 1 & 443 \\
$1,3,4,5,6,7$ & 4751 & 2 & 259 \\
$1,2,4,5,6,7$ & 4731 & 3 & 279 \\
$1,2,3,5,6,7$ & 3372 & 4 & 1638
\end{tabular}




$\begin{array}{llll}1,2,3,4,6,7 & 4088 & 5 & 922 \\ 1,2,3,4,5,7 & 4466 & 6 & 544 \\ 1,2,3,4,5,6 & 4085 & 7 & 925\end{array}$

Overall performance of the 7 layers WF-CNN in the entire 6 hours $\quad \mathbf{9 8 . 0 7} \pm \mathbf{1 . 9 2}$

Table S7. Results of the WF-CNN classifier for post-HI spike transient identification in experimental data (entire 6 hours -5 layers)

\begin{tabular}{llll}
\hline Trained and validated on Sheep No. & No. of patterns in the train dataset & Tested on Sheep No. & No. of pat \\
$2,3,4,5,6,7$ & 4567 & 1 & 443 \\
$1,3,4,5,6,7$ & 4751 & 2 & 259 \\
$1,2,4,5,6,7$ & 4731 & 3 & 279 \\
$1,2,3,5,6,7$ & 3372 & 4 & 1638 \\
$1,2,3,4,6,7$ & 4088 & 5 & 922 \\
$1,2,3,4,5,7$ & 4466 & 6 & 544 \\
$1,2,3,4,5,6$ & 4085 & 7 & 925 \\
\hline
\end{tabular}

Overall performance of the 5 layers WF-CNN in the entire 6 hours $\quad \mathbf{9 7 . 9 6} \pm \mathbf{1 . 4 8}$ 

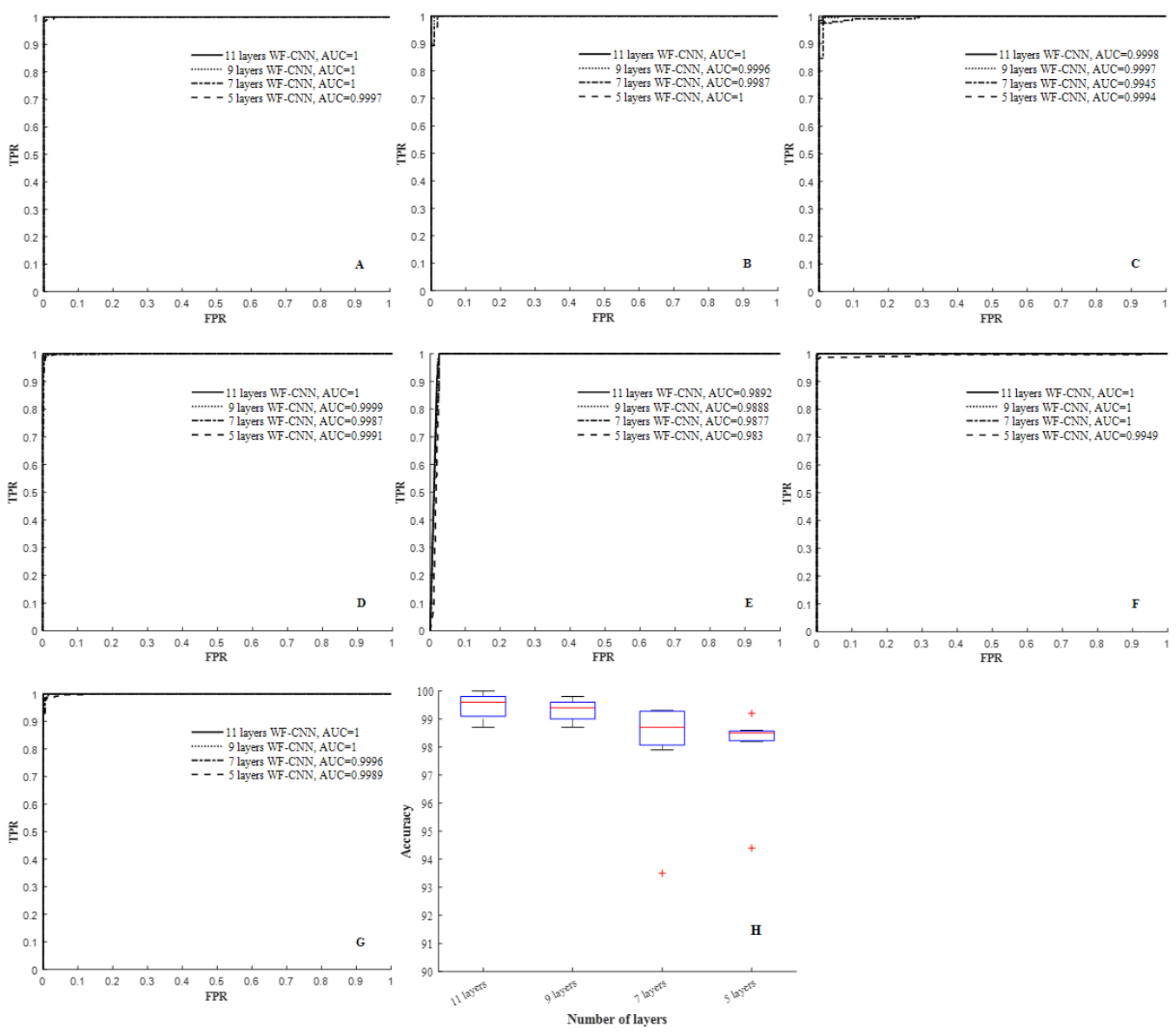

Figure 1: (A) to $(\mathrm{G})$ : ROC curves and the corresponding AUC values from 7-fold crossvalidation of the results along 6 hours of $1024 \mathrm{~Hz}$ data across 7 preterm fetal sheep (42 hours total) using 11, 9, 7 and 5 layers in the proposed WF-CNN classifier. The data for each WF-CNN classifier are presented as mean \pm SD in the boxplot $(\mathrm{H})$ demonstrating reduced accuracy and increased variability with fewer layers.

\section{Appendix C}

Table S8. Results of the 1D-CNN classifier for post-HI spike transient identification in experimental data (entire 6 hours - 11 layers)

Trained and validated on Sheep No. No. of patterns in the train dataset Tested on Sheep No. No. of pat 
$2,3,4,5,6,7$

$1,3,4,5,6,7$

$1,2,4,5,6,7$

$1,2,3,5,6,7$

$1,2,3,4,6,7$

$1,2,3,4,5,7$

$1,2,3,4,5,6$
4567

4751

4731

3372

4088

4466

4085

\section{1}

2

3

4

5

6

7
443

259

279

1638

922

544

925

Overall performance of the 11-layers $1 \mathrm{D}-\mathrm{CNN}$ in the entire 6 hours $\quad \mathbf{9 9 . 2 7 \pm 0 . 5 1}$

Table S9. Results of the 1D-CNN classifier for post-HI spike transient identification in experimental data (entire 6 hours - 9 layers)

\begin{tabular}{llll}
\hline Trained and validated on Sheep No. & No. of patterns in the train dataset & Tested on Sheep No. & No. of pat \\
$2,3,4,5,6,7$ & 4567 & 1 & 443 \\
$1,3,4,5,6,7$ & 4751 & 2 & 259 \\
$1,2,4,5,6,7$ & 4731 & 3 & 279 \\
$1,2,3,5,6,7$ & 3372 & 4 & 1638 \\
$1,2,3,4,6,7$ & 4088 & 5 & 922 \\
$1,2,3,4,5,7$ & 4466 & 6 & 544 \\
$1,2,3,4,5,6$ & 4085 & 7 & 925 \\
\hline
\end{tabular}

Overall performance of the 9 layers

98.07 \pm 2.63

$1 \mathrm{D}-\mathrm{CNN}$ in the entire 6 hours

Table S10. Results of the 1D-CNN classifier for post-HI spike transient identification in experimental data (entire 6 hours -7 layers)

\begin{tabular}{llll}
\hline Trained and validated on Sheep No. & No. of patterns in the train dataset & Tested on Sheep No. & No. of pat \\
$2,3,4,5,6,7$ & 4567 & 1 & 443 \\
$1,3,4,5,6,7$ & 4751 & 2 & 259 \\
$1,2,4,5,6,7$ & 4731 & 3 & 279 \\
$1,2,3,5,6,7$ & 3372 & 4 & 1638
\end{tabular}


$1,2,3,4,6,7$

$1 \mathrm{D}-\mathrm{CNN}$ in the entire 6 hours

Table S11. Results of the 1D-CNN classifier for post-HI spike transient identification in experimental data (entire 6 hours -5 layers)

\begin{tabular}{llll} 
Trained and validated on Sheep No. & $\begin{array}{l}\text { No. of patterns in the train dataset } \\
2,3,4,5,6,7\end{array}$ & Tested on Sheep No. & No. of pat \\
$1,3,4,5,6,7$ & 4567 & 1 & 443 \\
$1,2,4,5,6,7$ & 4751 & 2 & 259 \\
$1,2,3,5,6,7$ & 4731 & 3 & 279 \\
$1,2,3,4,6,7$ & 3372 & 4 & 1638 \\
$1,2,3,4,5,7$ & 4088 & 5 & 922 \\
$1,2,3,4,5,6$ & 4466 & 6 & 544 \\
\hline
\end{tabular}

Overall performance of the 5 layers $1 \mathrm{D}-\mathrm{CNN}$ in the entire 6 hours $\quad \mathbf{9 5 . 8 6} \pm \mathbf{3 . 7 4}$ 

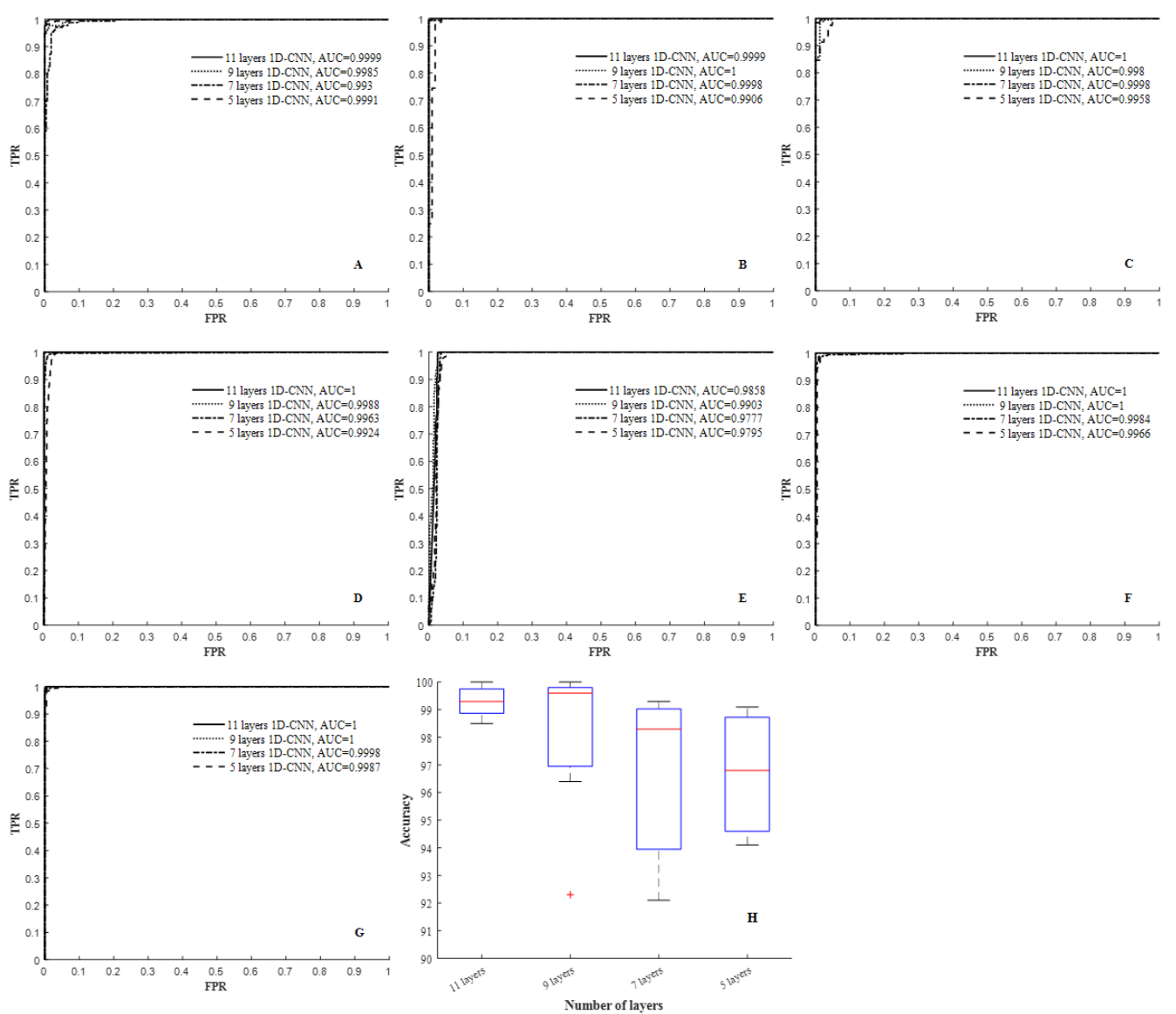

Figure 2: (A) to (G): ROC curves and the corresponding AUC values from 7-fold crossvalidation of the results along 6 hours of $1024 \mathrm{~Hz}$ data across 7 preterm fetal sheep (42 hours total) using 11, 9, 7 and 5 layers in the 1D-CNN classifier. The data for each 1D-CNN classifier are presented as mean $\pm \mathrm{SD}$ in the boxplot $(\mathrm{H})$ demonstrating reduced accuracy and increased variability with fewer layers.

\section{Appendix D}

Table S12. Results of the wavelet-Type-I-Fuzzy classifier for post-HI spike transient identification in experimental data (entire 6 hours)

Tested on Sheep No. No. of patterns in the Test-set TP hits FP hits FN hits Sensitivity [\%] Selecti 


\begin{tabular}{lllllll}
1 & 443 & 441 & 3 & 2 & 99.55 & 99.32 \\
2 & 259 & 258 & 4 & 1 & 99.61 & 98.47 \\
3 & 279 & 275 & 8 & 4 & 98.57 & 97.17 \\
4 & 1638 & 1621 & 9 & 17 & 98.96 & 99.45 \\
5 & 922 & 916 & 6 & 6 & 99.35 & 99.35 \\
6 & 544 & 536 & 5 & 8 & 98.53 & 99.08 \\
7 & 925 & 921 & 4 & 4 & 99.57 & 99.57 \\
\hline
\end{tabular}

Overall performance of the WT-Type-I-Fuzzy in the entire 6 hours $\quad \mathbf{9 9 . 0 4} \pm \mathbf{0 . 5 3}$

Table S13. Results of the FFT-Type-I-Fuzzy classifier for post-HI spike transient identification in experimental data (entire 6 hours)

\begin{tabular}{lllllll}
\hline Tested on Sheep No. & No. of patterns in the Test-set & TP hits & FP hits & FN hits & Sensitivity [\%] & Selecti \\
1 & 443 & 438 & 6 & 5 & 98.87 & 98.65 \\
2 & 259 & 254 & 7 & 5 & 98.07 & 97.32 \\
3 & 279 & 272 & 9 & 7 & 97.49 & 96.80 \\
4 & 1638 & 1615 & 13 & 23 & 98.60 & 99.20 \\
5 & 922 & 911 & 11 & 11 & 98.81 & 98.81 \\
6 & 544 & 534 & 9 & 10 & 98.16 & 98.34 \\
7 & 925 & 921 & 8 & 4 & 99.57 & 99.14 \\
\hline
\end{tabular}

Overall performance of the FFT-Type-I-Fuzzy in the entire 6 hours $\quad \mathbf{9 8 . 4 2 \pm 0 . 7 1}$

Table S14. The architecture of the proposed deep WF-CNN classifier

\begin{tabular}{|c|c|c|c|c|c|c|}
\hline Layers & Type & No. of Neurons (Output layer) & Kernel size & Stride & Padding & No. of \\
\hline $0-1$ & Conv. & $72 \times 3$ & {$\left[\begin{array}{ll}3 & 3\end{array}\right]$} & 1 & 1 & 32 \\
\hline $1-2$ & Max_pool & $36 \times 2$ & {$\left[\begin{array}{ll}2 & 1\end{array}\right]$} & 2 & 0 & \\
\hline $2-3$ & Conv. & $36 \times 2$ & {$\left[\begin{array}{ll}3 & 3\end{array}\right]$} & 1 & 1 & 64 \\
\hline $3-4$ & Max_pool & $18 \times 1$ & {$\left[\begin{array}{ll}2 & 2\end{array}\right]$} & 2 & 0 & \\
\hline $4-5$ & Conv. & $18 \times 1$ & {$\left[\begin{array}{ll}3 & 3\end{array}\right]$} & 1 & 1 & 128 \\
\hline $5-6$ & Max_pool & $9 \times 1$ & {$\left[\begin{array}{ll}2 & 1\end{array}\right]$} & 2 & 0 & \\
\hline
\end{tabular}




\begin{tabular}{|c|c|c|c|c|c|}
\hline $6-7$ & Conv. & $9 \times 1$ & {$\left[\begin{array}{ll}3 & 3\end{array}\right]$} & 1 & 1 \\
\hline $7-8$ & Max_pool & $4 \times 1$ & {$\left[\begin{array}{ll}3 & 1\end{array}\right]$} & 2 & 0 \\
\hline \multirow[t]{3}{*}{$9-11$} & Fully_connected & 1280 & & & \\
\hline & Fully_connected & 20 & & & \\
\hline & Fully_connected & 2 & & & \\
\hline Output & Softmax \&Classi & & & & \\
\hline
\end{tabular}

Table S15. The architecture of the proposed deep 1D-CNN classifier

\begin{tabular}{|c|c|c|c|c|c|c|}
\hline Layers & Type & $\begin{array}{l}\text { No. of } \\
\text { Neurons } \\
\text { (Output } \\
\text { layer) }\end{array}$ & Kernel size & Stride & Padding & $\begin{array}{l}\text { No. of } \\
\text { Filters }\end{array}$ \\
\hline $0-1$ & Conv. & $72 \times 1$ & {$\left[\begin{array}{ll}3 & 1\end{array}\right]$} & 1 & 0 & 4 \\
\hline $1-2$ & Max_pool & $70 \times 1$ & {$\left[\begin{array}{ll}2 & 1\end{array}\right]$} & 2 & 0 & \\
\hline $2-3$ & Conv. & $35 \times 1$ & {$\left[\begin{array}{ll}3 & 1\end{array}\right]$} & 1 & 0 & 6 \\
\hline $3-4$ & Max_pool & $33 \times 1$ & {$\left[\begin{array}{ll}3 & 1\end{array}\right]$} & 2 & 0 & \\
\hline $4-5$ & Conv. & $16 \times 1$ & {$\left[\begin{array}{ll}3 & 1\end{array}\right]$} & 1 & 0 & 8 \\
\hline $5-6$ & Max_pool & $14 \times 1$ & {$\left[\begin{array}{ll}2 & 1\end{array}\right]$} & 2 & 0 & \\
\hline $6-7$ & Conv. & $7 \times 1$ & {$\left[\begin{array}{ll}3 & 1\end{array}\right]$} & 1 & 0 & 10 \\
\hline $7-8$ & Max_pool & $5 \times 1$ & {$\left[\begin{array}{ll}3 & 1\end{array}\right]$} & 2 & 0 & \\
\hline \multirow[t]{3}{*}{$9-11$} & $\begin{array}{l}\text { Fully_- } \\
\text { connected }\end{array}$ & 40 & & & & \\
\hline & $\begin{array}{l}\text { Fully_- } \\
\text { connected }\end{array}$ & 10 & & & & \\
\hline & $\begin{array}{l}\text { Fully_- } \\
\text { connected }\end{array}$ & 2 & & & & \\
\hline \multirow[t]{2}{*}{ Output } & Softmax \& & & & & & \\
\hline & Classification & & & & & \\
\hline
\end{tabular}

\section{Appendix F}



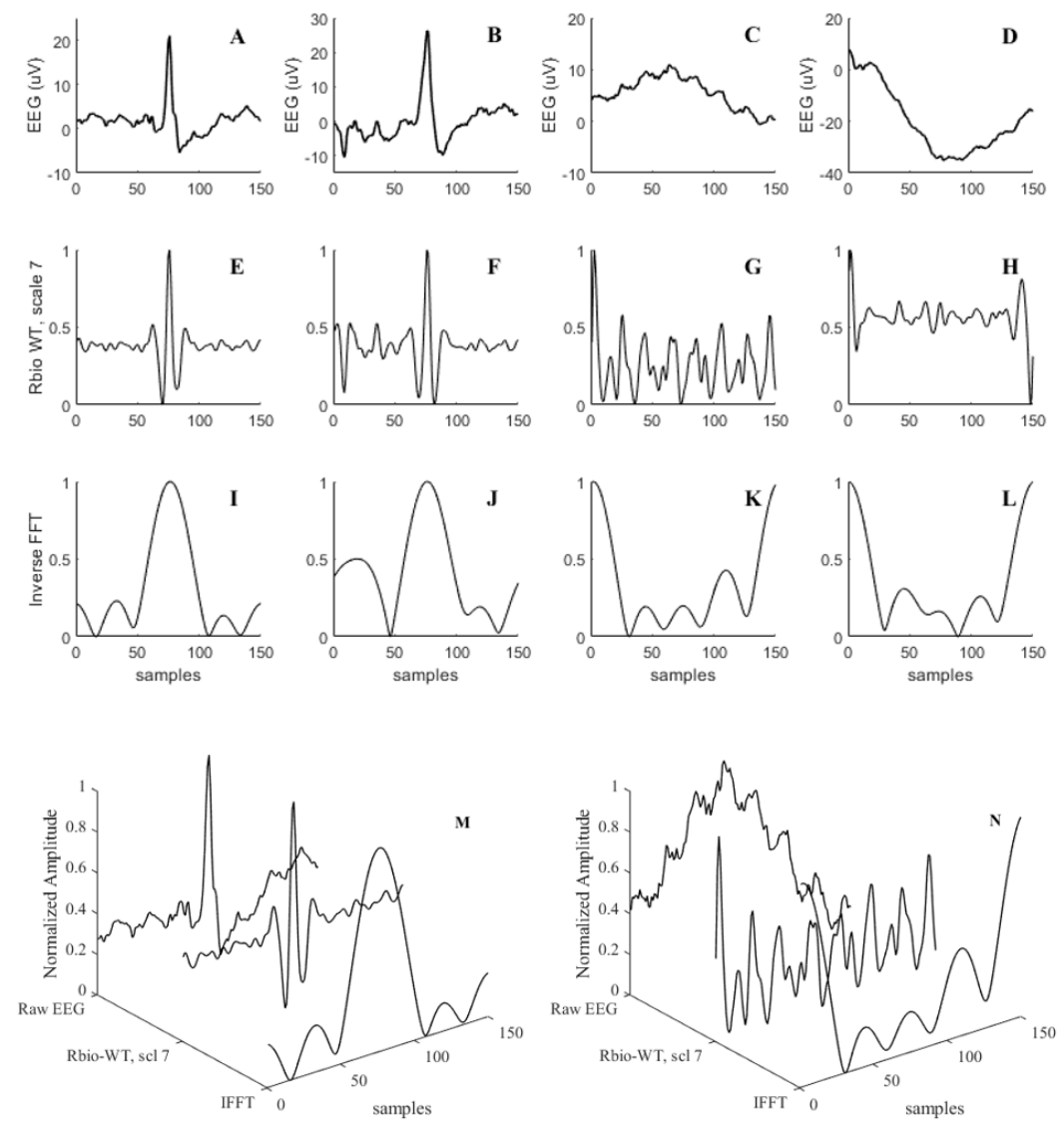

Figure 3: Examples of post-HI micro-scale ECoG spike transients (A, B) and non-spike ECoG background $(\mathrm{C}, \mathrm{D})$. The corresponding reverse biorthogonal wavelet transforms (rbio2.8) of the spikes (E, F) and non-spikes $(\mathrm{G}, \mathrm{H})$ at scale 7. The corresponding inverse Fourier transforms of the spikes (I, J) and non-spikes (K, L) using band-pass filter 80-120 $\mathrm{Hz}$. The combination of these three time-series (to form a matrix of size $72 \times 1 \times 3$ ) was used for training and validation of the WF-CNN classifier $(\mathrm{M}, \mathrm{N})$. 

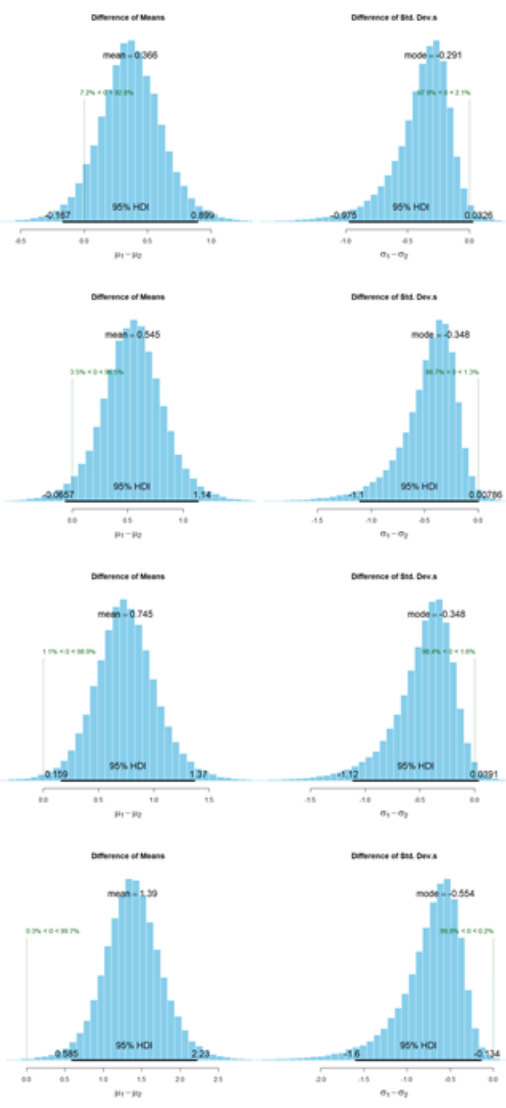
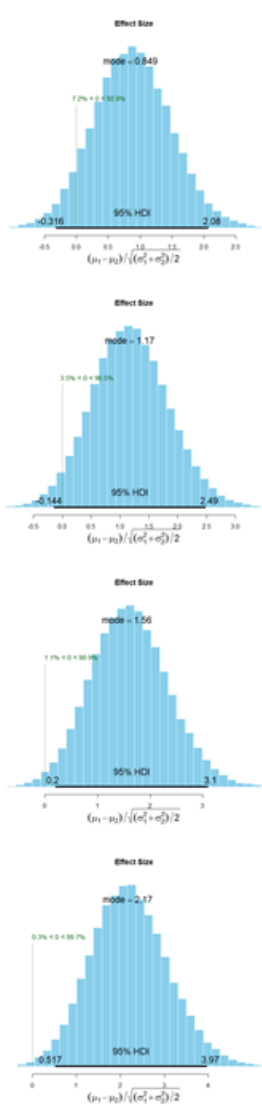

Figure 4: Bayesian estimation analysis for (A) 17-layers WS-CNN vs 11-layers WF-CNN (B) 17-layers WS-CNN vs 11-layers 1D-CNN (C) 17-layers WS-CNN vs WT-Fuzzy (D) 17-layers WS-CNN vs FFT-Fuzzy. Results demonstrate how the deepest WS-CNN could statistically outperform the other classifiers. 


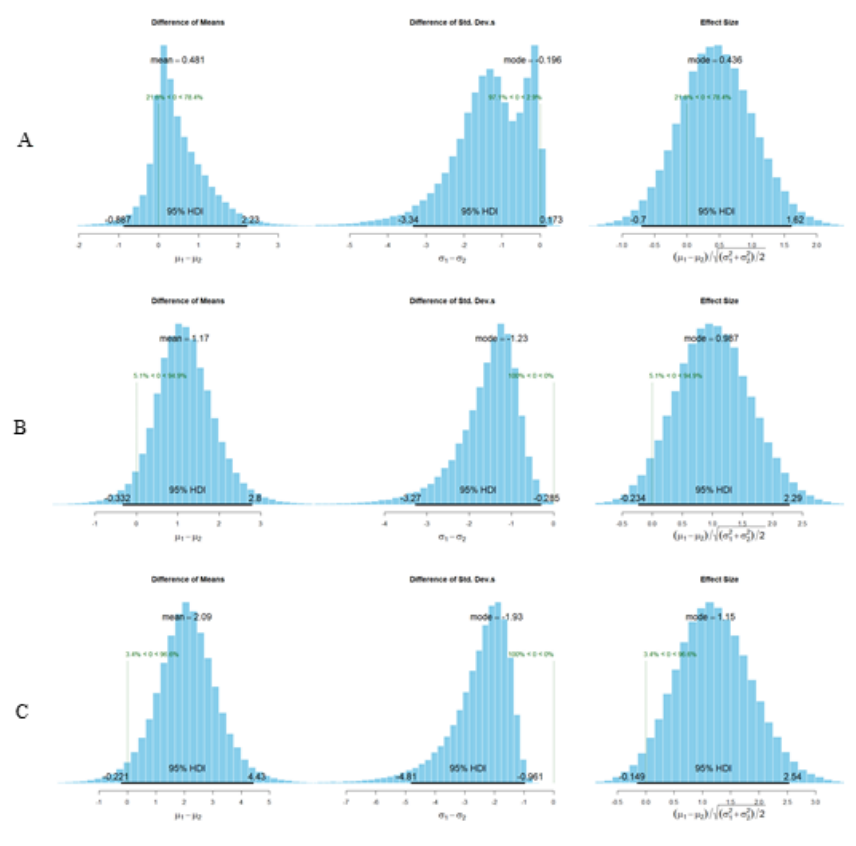

Figure 5: Bayesian estimation analysis for (A) 17-layers WS-CNN vs 13-layers WS-CNN (B) 17-layers WS-CNN vs 9-layers WS-CNN (C) 17-layers WS-CNN vs 5-layers WSCNN. Results demonstrate how the deepest WS-CNN could statistically outperform other WS-CNN classifiers with shallower structures. 